\section{A) Check for updates}

Cite this: Food Funct., 2022, 13, 3746

\title{
Gut microbiota can utilize prebiotic birch glucuronoxylan in production of short-chain fatty acids in rats $\uparrow$
}

\author{
Emma Kynkäänniemi, (D) *a Maarit H. Lahtinen, (D) a Ching Jian, ${ }^{\mathrm{b}}$ Anne Salonen, (D) ${ }^{\mathrm{b}}$ \\ Timo Hatanpää, (iD c Kirsi S. Mikkonen (iD a,d and Anne-Maria Pajari iD a
}

\begin{abstract}
Birch-derived glucuronoxylan (GX)-rich hemicellulose extract is an abundantly available by-product of the forest industry. It has multifunctional food stabilizing properties, and is rich in fiber and polyphenols. Here, we studied its effects on colonic metabolism and gut microbiota in healthy rats. Male and female Wistar rats $(n=42)$ were fed AIN-93G-based diets with $10 \%(w / w)$ of either cellulose (control), a polyphenol and GX-rich extract (GXpoly), or a highly purified GX-rich extract (pureGX) for four weeks. Both the GXpoly and pureGX diets resulted in changes on the gut microbiota, especially in a higher abundance of Bifidobacteriaceae than the cellulose containing diet $(p<0.001)$. This coincided with higher concentrations of microbial metabolites in the luminal contents of the GX-fed than control rats, such as total short-chain fatty acids (SCFAs) ( $p<0.001)$, acetate $(p<0.001)$, and N-nitroso compounds (NOCs) $(p=$ 0.001). The difference in the concentration of NOCs was not seen when adjusted with fecal weight. GX supplementation supported the normal growth of the rats. Our results indicate that GXpoly and pureGX can favorably affect colonic metabolism and the gut microbiota. They have high potential to be used as prebiotic stabilizers to support more ecologically sustainable food production.
\end{abstract}

Received 18th November 2021 Accepted 6th March 2022

DOI: $10.1039 /$ d1fo03922a rsc.li/food-function have been widely studied for their health-promoting effects, including better gut health, ${ }^{7,8}$ little is known about the health effects of wood-derived glucuronoxylan.

The extraction process of fibers can have an effect on their sidechains, ${ }^{9}$ co-component content of extracts, ${ }^{10}$ and bonds between components. ${ }^{11}$ Depending on the processing method, birch-derived GX-rich extracts can contain varying amounts of lignin and lignin residues. ${ }^{5}$ Lignin is a polyphenolic macromolecule with a complex chemical structure, ${ }^{4}$ which is thought to stay intact in the digestive tract without being utilized by the intestinal bacteria. ${ }^{12}$ However, there is evidence that the rat intestinal microbes can metabolize dietary lignin into the mammalian lignans enterolactone and enterodiol, ${ }^{13}$ which have shown to have anticarcinogenic and anti-atherosclerotic effects in animal models as well as in population studies. ${ }^{14}$ In addition, some in vitro studies have shown that the human fecal microbes may partially metabolize lignin at a slow rate. $^{15,16}$ By comparing differently processed GX with varying amount of lignin, the effects of lignin utilization by the microbes on shaping the compositions of the gut microbiota and its metabolites can be understood.

Many of the health benefits of dietary fibers are mediated by the microbes residing in the gastrointestinal tract, known as the gut microbiota. ${ }^{17}$ Previous studies have demonstrated the prebiotic properties of certain wood-derived fibers, such as 
arabinoglucuronoxylan (AGX), one of the main xylans in softwood, ${ }^{18}$ and xylo-oligosaccharides (XOS), carbohydrate oligomers extracted from xylan-containing lignocellulosic materials. ${ }^{18-21}$ In particular, they have stimulated the growth of Bifidobacterium and Lactobacillus spp., which are considered health-promoting gut microbes. Many human gut bacteria, especially some members of Firmicutes, Bacteroidetes, and genera Bifidobacterium, can ferment dietary fibers resulting in short-chain fatty acids (SCFAs). ${ }^{22}$ SCFAs may exert beneficial effects on host metabolism, gut immunity, and protect against colorectal cancer. ${ }^{23}$ In vitro and in vivo studies suggest that XOS increases acetate levels $\mathrm{s}^{24-28}$ and AGX is propiogenic. ${ }^{18} \mathrm{~A}$ recent study showed that alkali-extracted birchwood xylan induced butyrate production and anti-inflammatory effects in the co-culture of Bacteroides dorei and Cenarchaeum symbiosum. ${ }^{29}$ On the other hand, microbes can also produce potentially harmful metabolites such as $\mathrm{N}$-nitroso compounds (NOCs) $^{30}$ and branched-chain fatty acids (BCFAs) via, e.g., protein fermentation. ${ }^{31}$ Some dietary fibers have shown to decrease concentration of NOCs ${ }^{32,33}$ and BCFAs ${ }^{34}$ in stool, but the effects of GX on the potentially harmful microbial metabolites remain unclear. Moreover, the ability of gut bacteria to metabolize differently processed xylans, despite their similarity in structure, seem to differ. ${ }^{35,36}$ Taken together, little is known regarding the effects of wood-derived GX on the gut microbiota and its metabolites in vivo, as the few available studies were based on in vitro fermentation. ${ }^{29}$

The current study aimed to evaluate the impact of previously unused birch wood extracts on colon metabolism and gut microbiota in healthy rats. We investigated the effects of feeding a polyphenol-and GX-rich extract or a highly purified GX extract on the gut microbiota and the production of SCFAs and NOCs in rats. This study highlights the dietary potential of an abundantly available, but previously unused birch extract as a valuable source of dietary fiber and bioactive compounds.

\section{Methods}

\subsection{Glucuronoxylan-rich hemicellulose extracts}

The GX- and polyphenol-rich extract (GXpoly) was obtained from the Natural Resources Institute Finland (Luke) and was prepared via pressurized hot water extraction, according to Kilpeläinen et al. ${ }^{37}$ and spray dried using a Buchi Mini Spray Dryer B-290 (Buchi, Switzerland). Spray drying was carried out at an inlet temperature of $170{ }^{\circ} \mathrm{C}$, an outlet temperature of $65{ }^{\circ} \mathrm{C}$, and a drying air flow rate of $667 \mathrm{~L} \mathrm{~h}^{-1}$. The highly purified GX-rich extract (pureGX) was obtained from $\mathrm{CH}$-Bioforce Oy. The extract was purified from xylan concentrate $(20 \% \mathrm{w} / \mathrm{w})$ using BLN-process. ${ }^{38}$ Briefly, the concentrate was slowly mixed with $95 \%$ ethanol and left to settle overnight. The supernatant was removed, added to ethanol, mixed, and again left to settle overnight. This precipitation was then repeated once. The remaining precipitate was drained and air-dried for a few hours in a fume hood. After this, the precipitate was dried in a vacuum oven at $55{ }^{\circ} \mathrm{C}$ for the next four days and then ground into powder. The main carbohydrate in both extracts was $\beta$-Dxylopyranosyl. In addition, extracts, especially GXpoly, contained polyphenolic compounds like lignin. The chemical structure of the extracts has been previously characterized by Mikkonen et al. ${ }^{39}$ (Table S1 $\dagger$ ).

\subsection{Animals}

The animal study protocol was approved by the Finnish National Animal Experiment Board (Eläinkoelautakunta, ELLA; permit code: ESAVI/12806/2019), and the research was carried out according to ARRIVE (Animal Research: Reporting of In Vivo Experiments) guidelines. ${ }^{40}$ Four-week-old male and female Wistar (RccHan: WIST) rats were purchased from Envigo Netherlands (Horst, The Netherlands) and used for the study after 12 days of acclimatization, during which the rats were on a SDS RM1 diet (Special Diets Services, Witham, UK). After the acclimatization, the rats were randomized into three body weight-matched groups, seven males and seven females in every group, altogether 14 animals per dietary group. They were housed in plastic IVC-cages, 3 or 4 rats per cage, on Nestlets bedding (Oy BN-Technology Ab, Helsinki, Finland). They were fed a pelleted, customized AIN-93G diet (Envigo Teklad Diets, Madison WI, The United States) for 28 days containing either $10 \%(\mathrm{w} / \mathrm{w})$ of cellulose (control group), $10 \%$ of polyphenol-and GX-rich extract (GXpoly), or 10\% of highly purified GX (pureGX) (Table 1). Rats had ad libitum access to water and feed. Their bodyweight was measured once a week. The temperature at the housing facility was maintained at $21 \pm$ $0.2{ }^{\circ} \mathrm{C}$ and relative humidity at $50 \pm 3 \%$, with a 12 -hour artificial light/dark cycle. In the third week of the study, rats were held in metabolic cages (3701M081, Tecniplast, Buguggiate, Italy) for 24 hours to measure the individual fecal weight ( $\mathrm{g}$ day $\left.^{-1}\right)$, food intake $\left(\mathrm{g}^{\text {day }}{ }^{-1}\right)$, and water intake $\left(\mathrm{mL}\right.$ day $\left.^{-1}\right)$. At the end of the four-week dietary treatment, the rats were killed using $\mathrm{CO}_{2}$ inhalation, and the cecum and colon contents were

Table 1 Ingredient composition of the experimental diets

\begin{tabular}{|c|c|c|c|}
\hline Diets & Control & GXpoly & pureGX \\
\hline \multicolumn{4}{|l|}{ Ingredient ( $\mathrm{g} \mathrm{kg}^{-1}$ of diet) } \\
\hline Casein & 200 & 200 & 200 \\
\hline L-Cystine & 3 & 3 & 3 \\
\hline Corn starch & 342 & 342 & 342 \\
\hline Maltodextrin & 132 & 132 & 132 \\
\hline Sucrose & 100 & 100 & 100 \\
\hline Soybean oil & 70 & 70 & 70 \\
\hline Mineral Mix, AIN-93G-MX & 35 & 35 & 35 \\
\hline Vitamin Mix, AIN-93-VX & 15 & 15 & 15 \\
\hline Vitamin $\mathrm{K} 1$, phylloquinone & 0.002 & 0.002 & 0.002 \\
\hline TBHQ, antioxidant & 0.014 & 0.014 & 0.014 \\
\hline Cellulose & 100 & 0 & 0 \\
\hline GXpoly $^{a}$ & 0 & 100 & 0 \\
\hline pureGX $^{b}$ & 0 & 0 & 100 \\
\hline
\end{tabular}

All diets were isocaloric $\left(3.6 \mathrm{kcal} \mathrm{g}^{-1}\right.$ ) and contained $17.7 \%$ protein, $7.2 \%$ fat, and $55.6 \%$ carbohydrate. ${ }^{a}$ Glucuronoxylan- and polyphenolrich hemicellulose extract. ${ }^{b}$ Highly purified glucuronoxylan-rich hemicellulose extract. 
collected. The cecal and fecal samples were kept at the maximum three hours at $4{ }^{\circ} \mathrm{C}$ until frozen at $-70{ }^{\circ} \mathrm{C}$.

\subsection{Lignin content analysis of GX-rich extracts and diets with} pyrolysis-gas chromatography/mass spectrometry (Pyr-GC/MS)

Samples of GX-rich extracts and diets were analyzed using EGA/PY-3030D Multi-functional Pyrolyzer (Frontier Laboratories Ltd, Japan) connected to a GCMS-QP2010 SE GC-MS (Shimadzu, Japan) using previously described method. ${ }^{5}$ All samples were analyzed in duplicates $(n=2)$. The sample size was approximately 100-150 $\mu \mathrm{g}$, and the samples were weighed and measured in $80 \mu \mathrm{L}$ cups. The pyrolysis was performed at $580{ }^{\circ} \mathrm{C}$ for $12 \mathrm{~s}$. The injection was performed using a direct mode at $300{ }^{\circ} \mathrm{C}$, which was the same as the pyrolyzer-GC interface. The carrier gas (Helium) was used at a flow rate of $1 \mathrm{~mL} \mathrm{~min}^{-1}$. The pyrolysis products were separated using an Ultra Alloy capillary column (length $30 \mathrm{~m}$, diameter $0.25 \mathrm{~mm}$, film thickness $0.25 \mu \mathrm{m}$, Frontier Laboratories Ltd, Japan). The initial temperature of the column oven was $50{ }^{\circ} \mathrm{C}$, which was increased at a rate of $10{ }^{\circ} \mathrm{C} \min ^{-1}$ to $320^{\circ} \mathrm{C}$ and then maintained for $9 \mathrm{~min}$, resulting in a 36 min total run time. The ion source temperature was $220^{\circ} \mathrm{C}$ and GC-MS interface temperature $320^{\circ} \mathrm{C}$. Electron ionization voltage of $70 \mathrm{eV}$ was used and the detector voltage was set automatically according to the tuning result. The MS scan range was 45-450 $\mathrm{m} / \mathrm{z}$. The identification of the pyrolysis products was based on the National Institute of Standards and Technology (NIST) database. Chromatograms were processed using the GCMS solution software, which was used to integrate 100 peaks for the hemicellulose and 120 peaks for the diet samples. The amount of lignin guaiacyl (G) and syringyl (S) units were calculated from the areas of identified 2-methoxyphenol-based and 2,6-dimethoxyphenol-based pyrolysis products.

\subsection{Dry-matter content analysis}

Dry matter content of 18 rat fecal samples ( $n=6$ per group, three females and males from each dietary group) from the proximal colon was determined with thermogravimetric analysis (TGA). TGA has been previously used to study the drying characteristics of fecal sludge, ${ }^{41}$ and the sensitivity of the method was an advantage when using small volumes of samples. The analyses were carried out on a Netzsch STA 449F3 Jupiter equipment (Netzsch-Gerätebau GmbH, Selb, Germany). Samples of 9-11 mg were heated in flowing nitrogen $\left(40 \mathrm{~mL} \mathrm{~min}^{-1}\right)$ atmosphere $(1 \mathrm{~atm})$ from $25-200{ }^{\circ} \mathrm{C}$ with a heating rate of $10^{\circ} \mathrm{C} \mathrm{min}^{-1}$. The weight loss was considered to be the water content and the residue the dry matter content of the sample.

\subsection{Microbiota analysis with 16S rRNA gene amplicon sequencing}

Bacterial DNA was extracted from ca.250 mg of cecal matter using the Repeated Bead Beating (RBB) method ${ }^{42}$ with the following modifications for automated DNA purification: $340 \mu \mathrm{L}$ and $145 \mu \mathrm{L}$ of lysis buffer was added to the first and second round of bead beating, respectively. $200 \mu \mathrm{L}$ of the clarified supernatant collected from the two bead beating rounds was used for DNA extraction with the Ambion Magmax ${ }^{\mathrm{TM}}$-96 DNA Multi-Sample Kit (4413022, Thermo Fisher Scientific, USA) using the KingFisherTM Flex automated purification system (ThermoFisher Scientific, USA). DNA was quantified using Quanti-iT ${ }^{\mathrm{TM}}$ Pico Green dsDNA Assay (Invitrogen, San Diego, CA, USA). Library preparation and Illumina MiSeq sequencing of the hypervariable V3-V4 regions of the 16S rRNA gene were performed as previously described. ${ }^{43}$ The sequencing data are available at the European Nucleotide Archive (ENA) under accession number PRJEB50937.

Sequences were processed using QIIME2 v.2020.11. pipeline. ${ }^{44}$ Demultiplexed 250 base paired-end sequences were denoised using DADA2 ${ }^{45}$ to obtain an amplicon sequence variant (ASV) table. Singletons (ASV present $<2$ times) and ASVs present in less than $10 \%$ of the samples were discarded. Taxonomic classification was performed using a pre-trained naive Bayes classifier implemented in QIIME2 against the SILVA 132 reference database. ${ }^{46}$ Samples meeting quality criteria $(n=35)$ had a mean sequencing depth of 20314 reads. Bacterial metagenome content was predicted from the $16 \mathrm{~S}$ rRNA gene-based microbial compositions. Functional inferences were made from the Kyoto Encyclopedia of Gene and Genomes (KEGG) catalog ${ }^{47}$ using PICRUSt2. ${ }^{48}$ Statistical analyses were performed using the $\mathrm{R}$ package mare ${ }^{49}$ To account for the varying sequencing depth, the number of reads per sample was used as an offset in all statistical models. Microbiota richness and Shannon diversity index were estimated using the vegan package. ${ }^{50}$ Overall microbiota structure was assessed using principal coordinate analysis (PCoA) on beta diversity computed using Bray-Curtis distances, representing the compositional dissimilarity between the samples. Permutational multivariate analysis of variance (PERMANOVA; adonis function in the vegan package ${ }^{50}$ ) with Bray-Curtis dissimilarities was used to identify factors contributing to variation in microbiota composition. Differential abundance for bacterial taxa or KEGG pathways between treatment groups was identified with the mare function "GroupTest" (based on generalized linear models using negative binomial distribution) accounting for sex and cage block. Associations between bacterial taxa and metabolites were assessed using Spearman's correlation. $P$-values were adjusted by the Benjamini-Hochberg method for multiple testing. FDRadjusted $p$-values $<0.05$ were considered statistically significant, though FDR-adjusted $p$-values $<0.1$ were also reported considering the small sample size in each treatment arm.

\subsection{Quantitative PCR for total bacteria}

Quantification of total bacteria was carried out by qPCR with the $331 \mathrm{~F} / 797 \mathrm{R}$ primers using a BioRad iCycler iQ thermal cycler system (BioRad, Hercules, CA) with HOT FIREPol ${ }^{\circ}$ EvaGreen ${ }^{\circledR}$ qPCR Mix Plus (Solis BioDyne, Tartu, Estonia) as described previously. ${ }^{51}$ The 10-log-fold standard curves ranging from $10^{2}$ to $10^{7}$ copies were produced using full-length amplicons of 16S rRNA gene of Bifidobacterium longum to convert the threshold cycle $(\mathrm{Ct})$ values into the average esti- 
mates of target bacterial genomes present in $1 \mathrm{~g}$ of feces (copy numbers per $\mathrm{g}$ of wet feces) in the assay. The qPCR assay was performed in triplicate. Precautions were taken to ensure that the data from each triplicate fell within 0.5 threshold cycle (Ct), and clear outliers ( $>2$ standard deviations) were removed before calculating the average $\mathrm{Ct}$ of each sample. Melting curves and non-template controls were used to assess run reliability. There was no detectable amplification arising from non-template controls in the assay. The amplification efficiency of the qPCR assay was 95\%. The absolute abundances were estimated and 16S rRNA gene copy-number corrected as previously described. ${ }^{51}$

\subsection{Nitroso compound analysis}

Nitroso compounds were measured from proximal colon fecal samples ( $n=12$ per group). Before the analysis, approximately $200 \mathrm{mg}$ fecal sample, $2 \mathrm{~mm}$ steel beads, and $1 \mathrm{~mL}$ HPLCgraded water per $200 \mathrm{mg}$ sample were disrupted with TissueLyser II (Qiagen, Hilden, Germany) for 10 minutes at 30 $1 \mathrm{~s}^{-1}$. The samples were then centrifuged (SL 8R, Thermo Scientific, Waltham, MA, USA) at $14000 \mathrm{~g}$ for 15 minutes at $4{ }^{\circ} \mathrm{C}$. The supernatant was collected and stored at $-80{ }^{\circ} \mathrm{C}$ until analysis.

The nitroso compounds analysis was modified from a previously described method. ${ }^{52}$ The samples were analyzed with Ecomedics CLD 88 Exhalyzer (Ecomedics, Dürnten, Switzerland) that uses chemiluminescence detection for measuring NO. For releasing the NO, the sample was injected into a purge vessel kept at $60{ }^{\circ} \mathrm{C}$ containing $15 \mathrm{~mL}$ tri-iodine solution ( $1 \mathrm{~g}$ potassium iodide (221945, Sigma Aldrich), $0.65 \mathrm{~g}$ iodine (196561000, Acros Organics), 20 mL HPLC-water, $70 \mathrm{~mL}$ acetic acid (20104.298, Analar Normapur), and antifoam emulsion (A6707, Sigma Aldrich). The purge vessel was connected via a condenser to a wash bottle maintained at $4{ }^{\circ} \mathrm{C}$ containing $15 \mathrm{~mL} 1 \mathrm{M} \mathrm{NaOH}$. The NO was transferred through the system to Ecomedics CLD 88 Exhalyzer via a $0.20 \mu \mathrm{m}$ polypropylene filter using helium (0-1 mbar) as a carrier gas. The NO values were detected with Chart v5.5.8 (eDAQ, Australia) that quantified the values of the sample based on the standardized values. The standardized values were produced by injecting $50 \mu \mathrm{L}$ of sodium nitrite (S2252, Sigma Aldrich) solution ten times in a concentration range of $0.1-10 \mathrm{pmol} \mu \mathrm{L}^{-1}$ into the purge vessel.

For determination of apparent total N-nitroso compounds (ATNC), $100 \mu \mathrm{L}$ supernatant was mixed with $100 \mu \mathrm{L} 0.1 \mathrm{M}$ $N$-ethylmaleimide (NEM) (E3876, Sigma Aldrich) and 0.01 M diethylenetriaminepentaacetic acid (DTPA) (D1133, Sigma Aldrich) in HPLC-water, and $500 \mu \mathrm{L}$ sulfanilamide (132851000, Acros Organics) solution (50 $\mathrm{g} \mathrm{l}^{-1}$ in $\mathrm{HCl}$ ), vortex mixed, incubated for 4 minutes and injected into the purge vessel. NEM and DTPA in a solution chelate metal ions and alkylate free thiol groups, thus preventing artifactual nitrosation. ${ }^{52,53}$ Sulfanilamide reacts with nitrite and forms a stable diazonium ion. ${ }^{54}$ The concentration of nitrosothiols (RSNO) was determined in the same way as ATNC with the addition of $100 \mu \mathrm{L}$ aqueous $10 \mathrm{mM} \mathrm{HgCl}_{2}$ (215465, Sigma Aldrich). Likewise, to determine the amount of nitrosyl iron (FeNO) compounds, the same solutions as for RSNO were used in addition to $100 \mu \mathrm{L}$ aqueous $10 \mathrm{mM} \mathrm{K} \mathrm{K}_{3} \mathrm{Fe}(\mathrm{CN})_{6}$ (196781000, Acros Organics). $\mathrm{HgCl}_{2}$ displaces $\mathrm{NO}^{+}$from $\mathrm{S}-\mathrm{NO},{ }^{54}$ and $\mathrm{K}_{3} \mathrm{Fe}(\mathrm{CN})_{6}$ oxidizes $\mathrm{Fe}^{2+}$ of FeNO to $\mathrm{Fe}^{3+} .{ }^{52}$ The value of RSNO was the difference between mercury(II) stable and unstable compounds, and the value of FeNO was the difference between ferricyanide stable and unstable compounds.

\subsection{Extraction and analysis of short-chain fatty acids}

Short-chain fatty acids were measured from cecal $(n=42)$ and distal colon fecal $(n=32$, control $=10$, GXpoly $=10$, pureGX $=$ 12) samples with a previously validated method based on gas chromatography with slight modifications. ${ }^{55}$ Briefly, the $200 \mathrm{mg}$ fecal and cecal samples were freeze-dried (Beta 2-8 LD Plus, Christ, Germany) for 48 hours at $-26^{\circ} \mathrm{C}$ and a vacuum pressure of 0.57 mbar. Approximately $20 \mathrm{mg}$ of Milliq water was added to fecal samples to facilitate drying.

Lyophilized samples $(10 \mathrm{mg})$ were mixed with $400 \mu \mathrm{L}$ of saturated $\mathrm{NaCl}, 10 \mu \mathrm{L}$ of $20 \mathrm{mM}$ 2-ethylbutyric acid (109959, Sigma Aldrich) was used as an internal standard, ${ }^{56}$ and $5 \mu \mathrm{L}$ of $2 \mathrm{mM} \mathrm{H}_{2} \mathrm{SO}_{4}$ solution was added to adjust the $\mathrm{pH}$ to 2 . Each sample was homogenized with $2 \mathrm{~mm}$ steel beads using Tissuelyser II for $2 \times 2$ minutes at $301 \mathrm{~s}^{-1}$. After homogenization, $400 \mu \mathrm{L}$ of pre-cooled EtOAc (34858, Honeywell) was added to sample tubes, vortex mixed for a minute, and centrifuged at $12000 \mathrm{rpm}$ for $10 \mathrm{~min}$ at $4{ }^{\circ} \mathrm{C}$. The supernatant was transferred to a $2 \mathrm{~mL}$ Eppendorf tube containing $0.25 \mathrm{~g}$ of anhydrous $\mathrm{Na}_{2} \mathrm{SO}_{4}$, centrifuged again, and then transferred to a gas chromatography vial for further analysis. A standard stock solution was prepared in ethyl acetate using similar SCFA concentrations as before: ${ }^{56} 460 \mathrm{mM}$ acetic acid (20104.298, Analar Normapur), $400 \mathrm{mM}$ propionic acid (W292400, Sigma Aldrich), 400 mM n-butyric acid (W222100, Sigma Aldrich), $100 \mathrm{mM}$ isobutyric acid (I1754, Sigma Aldrich), $200 \mathrm{mM}$ pentanoic acid (240370, Sigma Aldrich), $200 \mathrm{mM}$ isovaleric acid (129542, Sigma Aldrich). Calibration curves were obtained using 8 concentrations by diluting the stock solution from $1 / 20$ to $1 / 4000(\mathrm{v} / \mathrm{v})$. All calibration samples contained $20 \mathrm{mM}$ of the internal standard.

One microliter of the supernatant was injected into the gas chromatograph (Agilent 8890 GC System, Agilent Technologies, Santa Clara, CA, USA) equipped with Zebron ${ }^{\mathrm{TM}}$ ZB-FFAP, GC Cap. Column $30 \mathrm{~m} \times 0.32 \mathrm{~mm} \times 0.25 \mu \mathrm{m}$ (Phenomenex, Værløse, Denmark) with split ratio of $20: 1$. Initially, the oven temperature was maintained for a minute at $100{ }^{\circ} \mathrm{C}$, raised to $180{ }^{\circ} \mathrm{C}$ at $8^{\circ} \mathrm{C} \mathrm{min}^{-1}$, held for a minute and then increased to $200{ }^{\circ} \mathrm{C}$ at $20{ }^{\circ} \mathrm{C} \mathrm{min}{ }^{-1}$ and held there for $5 \mathrm{~min}$. Helium was used as a carrier gas at a flow rate of $1 \mathrm{~mL}$ $\mathrm{min}^{-1}$. SCFAs are expressed as micromole per gram dry weight.

\subsection{Statistics}

For non-sequencing data, the difference between the three dietary groups was analyzed with two-way ANOVA. Before the analysis, data were systematically explored to detect outliers, heterogeneity of variance, and non-normal distribution. Log 
transformation was used for variables that did not meet the assumptions of two-way ANOVA (ATNC, FeNO, RSNO, cecum and colon acetate, and colon butyrate). Results are reported as mean \pm standard deviation.

The cages of rats were included in statistical analysis by randomly dividing them into two blocks (7 rats per dietary treatment/block, both blocks containing both sexes). Two-way ANOVA was conducted using sex as a factor and block as a random factor. ANOVA model:

$$
X_{i j z}=\mu+\alpha_{i}+v_{j}+\gamma_{z}+\alpha v_{i j}+\varepsilon_{i j z}
$$

where $\alpha_{i}$ is the diet ( $i=$ control, GXpoly or pureGX); $v_{j}$ is sex ( $j=$ male or female); $\gamma_{z}$ is the random effect of block $(z=1$ or 2 ), and $\alpha v_{i j}$ is interaction between the diet and sex. $\varepsilon_{i j z}$ refers to residual error. For weight comparisons, the average body weight obtained during the feeding period was used as a dependent variable and initial weight as a covariate. The Tukey HSD test was used to compare the means of the groups. $P$-Value $\leq 0.05$ was considered significant. Analysis was conducted with IBM SPSS Statistics 25.0.

\section{Results}

\subsection{Lignin in the GXpoly diet}

To compare the polyphenol content of the differently processed GX and diets, we measured their lignin content using Pyr-GC/MS. ${ }^{5}$ As expected, the lignin content was higher in the GXpoly compared to the pureGX (Table 2). We also detected lignin in the GXpoly diet samples (Table 2) but not in the pureGX diet samples (not shown).

Table 2 Lignin content $^{a}$ (= syringyl (S) + guaiacyl (G), \%) of the GX extracts and the GXpoly diet based on area\% of lignin fragments ( $n=2$ per sample)

\begin{tabular}{lllll}
\hline Sample & G units (\%) & S units (\%) & S + G units (\%) & S/G ratio \\
\hline GXpoly $^{b}$ & $5.81 \pm 0.10$ & $12.22 \pm 2.16$ & $18.02 \pm 2.06$ & $2.11 \pm 0.40$ \\
pureGX $^{c}$ & $0.62 \pm 0.21$ & $1.72 \pm 0.42$ & $2.34 \pm 0.21$ & $3.06 \pm 1.72$ \\
GXpoly diet & N.D. & $0.67 \pm 0.03$ & $0.67 \pm 0.03$ & N.D.
\end{tabular}

${ }^{a}$ Measured using pyrolysis-gas chromatography/mass spectrometry.

${ }^{b}$ Glucuronoxylan- and polyphenol-rich hemicellulose extract. ${ }^{c}$ Highly purified glucuronoxylan-rich hemicellulose extract.

\subsection{Body weight, food intake and fecal dry-matter content did not differ between the control and GX-groups}

The rats gained weight normally during the feeding period, and no difference in the body weight gain was observed between the groups (Table 3 and Fig. S1†). Similarly, food intake did not vary across the groups. There was no statistically significant difference in the water intake and urine volume among the groups although the volumes were lowest in the pureGX group (Table 3).

To study the effects of GXpoly and pureGX on stool properties, we measured dry-matter content $(n=18)$ and weight of the fecal samples of the rats. Feeding the GX diets resulted in a similar dry-matter content of feces than the control diet (Table 3). However, fecal mass was significantly higher in the control group than in the pureGX, but not in the GXpoly, group (Table 3).

\subsection{GX alters cecal microbiota composition and predicted functions in healthy rats}

To assess the impact of the GX-enriched diets on dominant cecal bacterial communities, we utilized 16S rRNA gene amplicon sequencing and qPCR for total bacteria prepared from total cecal DNA. Strong clustering of the samples by the treatment group in principal coordinates analysis (PCoA) indicated significant differences in overall phylogenetic makeup between the two GX groups and the control group (Fig. 1A). The variance of the bacterial community compositions was predominantly explained by treatment $(40 \%, p=0.001$, Fig. 1A). Microbiota richness and alpha diversity estimated by the Shannon index were significantly lower in the GX groups than in the control group, while the total amount of bacteria, measured by qPCR, was significantly higher in GX groups ( $p<0.01$, Fig. 1B). No significant difference in microbiota richness, beta, or alpha diversity was found between the GXpoly and pureGX groups.

We next zoomed into individual bacterial taxa to identify specific bacteria that differed in abundance between the treatment groups (Fig. 2 and Table S2†). At the family level, the relative abundance of Bifidobacteriaceae was substantially enriched in the GX groups compared to the control group (estimated fold change $=150$ in GXpoly and 432 in pureGX, FDR- $p$ $<0.05$, Table $\mathrm{S} 2 \dagger)$. Other bacterial families that were significantly more abundant in both GX groups included an uncultured family of Rhodospirillales, Enterobacteriaceae, and Atopobiaceae (all FDR- $p<0.05$ ). The relative abundance of

Table 3 Effect of GX diets on weight gain, fecal weight, and dry-matter content, urine volume, and food and water intake in Wistar rats

\begin{tabular}{|c|c|c|c|c|}
\hline Diet & Control & GXpoly $^{a}$ & pureGX $^{b}$ & $p$-Value \\
\hline Weight gain (g) & $115.6 \pm 45.2$ & $116.4 \pm 45.2$ & $111.3 \pm 40.2$ & 0.661 \\
\hline Food intake $(\mathrm{g}) / 24 \mathrm{~h}$ & $17.2 \pm 2.9$ & $15.5 \pm 5.1$ & $15.1 \pm 4.7$ & 0.318 \\
\hline Water intake $(\mathrm{mL}) / 24 \mathrm{~h}$ & $32.9 \pm 12.0$ & $33.4 \pm 10.7$ & $25.0 \pm 11.6$ & 0.226 \\
\hline Urine volume $(\mathrm{mL}) / 24 \mathrm{~h}$ & $11.5 \pm 2.2$ & $11.3 \pm 2.4$ & $9.6 \pm 3.3$ & 0.100 \\
\hline Fecal weight $(\mathrm{g}) / 24 \mathrm{~h}$ & $3.2 \pm 1.1^{\mathrm{a}}$ & $2.2 \pm 2.8^{\mathrm{a}}$ & $1.3 \pm 1.1^{\mathrm{b}}$ & 0.031 \\
\hline Dry-matter content $\%(n=18)$ & $29.1 \pm 9.0$ & $23.3 \pm 5.8$ & $21.4 \pm 4.5$ & 0.176 \\
\hline
\end{tabular}

Statistically significant difference in group means labeled with different letters. ${ }^{a}$ Glucuronoxylan- and polyphenol-rich hemicellulose extract.

${ }^{b}$ Highly purified glucuronoxylan-rich hemicellulose extract. 
A

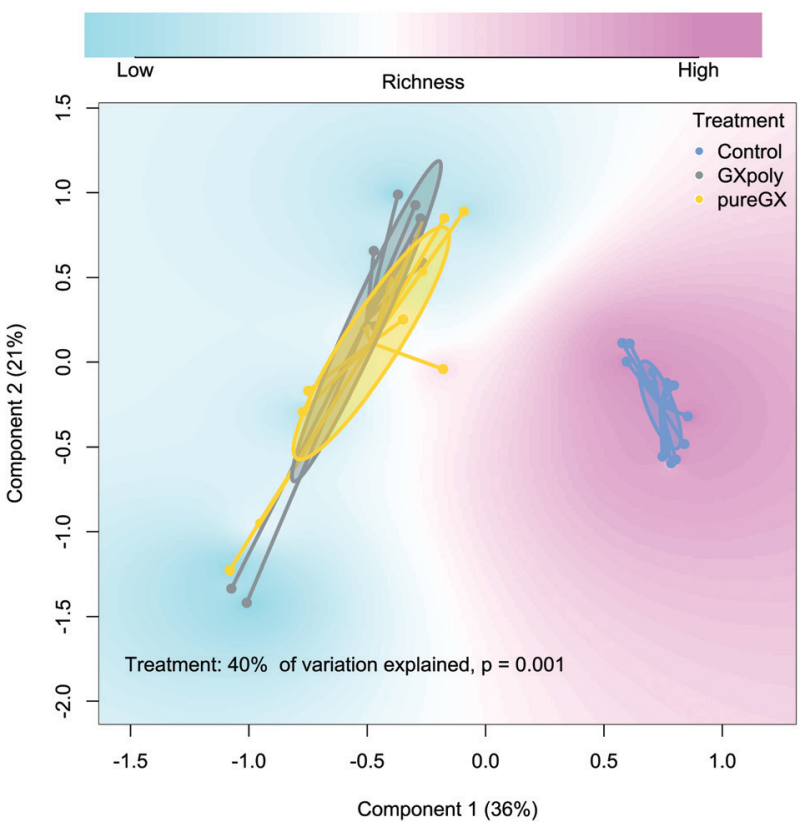

B

Treatment 审 Control 由x Gxoly 审 pureGX

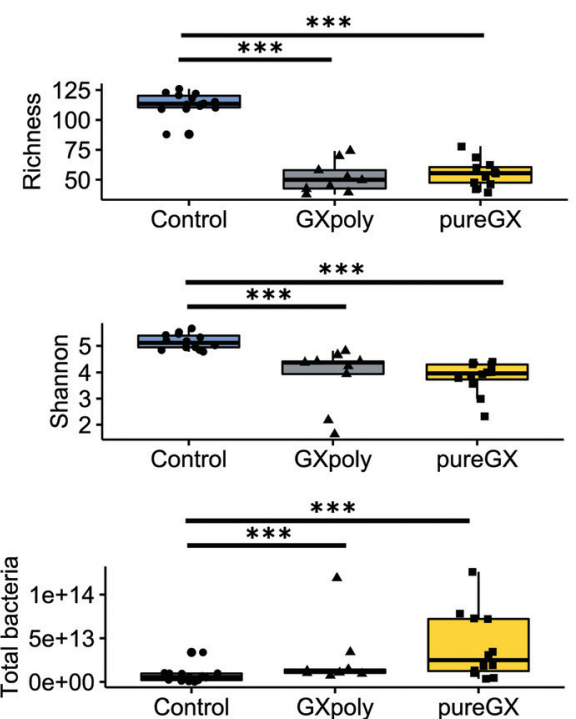

Fig. 1 Principal coordinates analysis (PCoA) plot of the gut microbiota from Wistar rats grouped by treatment with cellulose (control), GXpoly, or pureGX (A). Differences in microbiota richness, diversity (Shannon index) and total bacterial counts between three treatment groups (B). ${ }^{\star} p \leq 0.05$, ${ }^{* *} p \leq 0.01,{ }^{* * *} p \leq 0.001$, GXpoly = glucuronoxylan- and polyphenol-rich hemicellulose extract, pureGX = highly purified glucuronoxylan-rich hemicellulose extract.
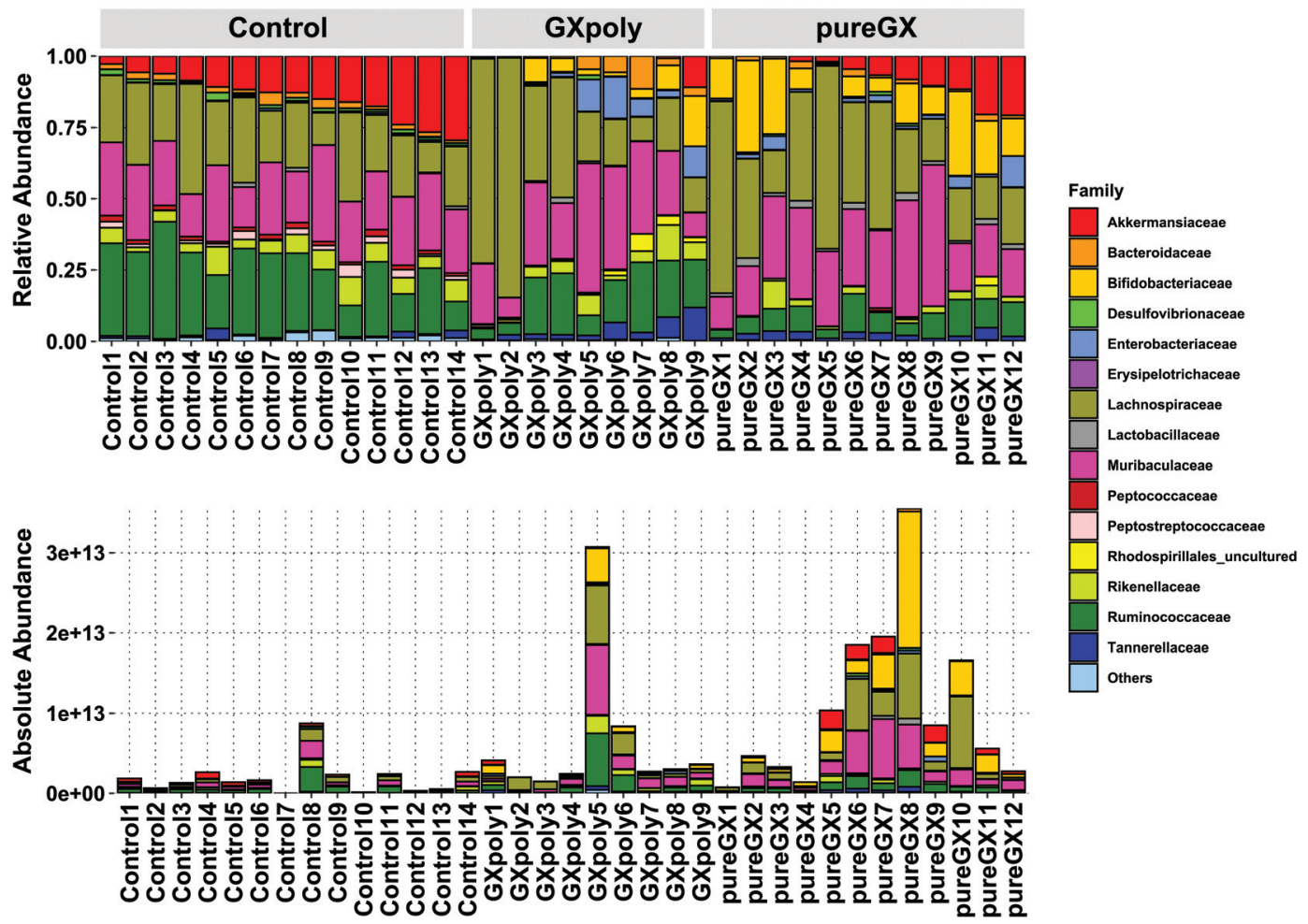

Fig. 2 Bar graphs show individual taxonomic profiles of gut bacteria at a family level in relative (upper panel) and absolute (lower panel) abundance in the Wistar rats fed either control (cellulose), GXpoly (glucuronoxylan- and polyphenol-rich hemicellulose extract), or pureGX (highly purified glucuronoxylan-rich hemicellulose extract) diet. 
Lactobacillaceae was selectively increased in the pureGX group (FDR- $p<0.05$ ). A 4-fold increase in polyphenol-degrading Eggerthellaceae was seen only in the GXpoly group in comparison to the control group (FDR- $p<0.05$ ). In contrast, the relative abundances of Peptococcaceae, Peptostreptococcaceae, and Desulfovibrionaceae were significantly reduced in both groups treated with GX (all FDR- $p<0.05$, Table S2†). Additionally, the GXpoly diet resulted in a depletion of Akkermansiaceae/ Akkermansia muciniphila (FDR-p < 0.05). At the genus level, in the GX groups, the most drastic enrichment was seen in Eisenbergiella, Bifidobacterium, and Flavonifractor, while the reduced proportion in the family Desulfovibrionaceae was attributed to the significant decrease in Bilophila (all FDR- $p<$ 0.05 , Table S $2 \dagger$ ). When absolute abundance was analyzed, the findings align well with the ones derived from relative abundance in most cases, with Bifidobacteriaceae showing the largest increase in abundance in the GX groups (Fig. 2 and Table $\mathrm{S} 2 \dagger$ ). In addition, absolute counts of Bacteroidaceae and Christensenellaceae were significantly higher in the GX groups (FDR- $p<0.05$ ), while the decreased absolute abundance of Desulfovibrionaceae failed to reach statistical significance (Table S2 $\dagger$ ). Some taxa exhibited significant differences in relative abundance between male and female rats mainly genera from family Ruminococcaceae and Lachnospiraceae (Table S3†).

To understand the functional implications of the observed taxonomic differences between the dietary groups, we inferred metagenomes using a computational approach that reconstructs functional composition of a metagenome connecting the sequenced genes to reference genomes. The predicted function that differed most between the groups was "lipopolysaccharide biosynthesis", which was ca. 50\% lower in the GXpoly group compared to the control group (Table S4†).

\subsection{Gut microbiota can utilize GX in production of microbial metabolites}

To see how the observed taxonomic difference between the dietary groups affected the fermentation in cecum and colon, we measured the concentration of known bacterial metabolites from cecal and fecal samples of the rats. Feeding GX led to significantly higher concentrations of ATNC $(p=0.001)$ and FeNO $(p<0.001)$ in the feces of GX-fed rats than in the control group (Fig. 3 and Table S5†). There was no significant difference in RSNO between the groups, and in five samples, the concentration of RSNO was below the detection limit.

The concentration of ATNC and FeNO were significantly higher in females than in males $(p=0.014$, and $p=0.041$ respectively, Table S5 $\dagger$ ). The concentrations of ATNC, RSNO, and FeNO correlated significantly with total bacterial counts ( $p$ $<0.001$, Fig. S2 $\dagger$ ). When the excretion of ATNC, FeNO, and RSNO was calculated per total fecal weight (the total excretion), it did not differ significantly between the groups (Table S6†).

GX feeding resulted in higher concentrations of acetic acid and total SCFAs compared to the controls in the cecum samples $(p<0.001$, Fig. 4A and Table S5 $\dagger)$. Conversely, the cecal concentrations of the BCFAs isobutyric and isovaleric acids were lower in the GX-fed groups than in the control group $(p<0.001$, Fig. 4A). The propionic acid concentration was highest in the pureGX-group with borderline statistical significance $(p=0.055)$. Even though the colonic concentrations of SCFAs did not reach a statistically significant difference between the groups (Fig. 4B), the trend was similar to the cecum. The concentrations of detected SCFAs correlated significantly with the absolute abundances of their respective producing bacteria, such as Bifidobacteriaceae that produce acetate, and Lactobacillaceae that produce acetate and propionate ( $p<0.05$, Fig. $\mathrm{S} 3 \dagger)$.

In addition to diet, sex affected cecum total SCFAs and acetate levels, which were higher in the females than in the males (Acetate: males: $152.2 \pm 57.1$; females: $213.9 \pm 89.4 ; p=$ 0.002; SCFA: males: $229.0 \pm 63.3$; females: $297.6 \pm 106.0 ; p=$ 0.004 ). Levels of both cecal (males: $7.0 \pm 1.6$; females: $6.3 \pm 1.6$; $p=0.045$ ) and colonic (males: $7.4 \pm 2.1$; females $5.3 \pm 2.3 ; p=$ $0.019)$ BCFAs were lower in the female rats than in the males. Body weight, food and water intake, and fecal characteristics by sex are presented in the Table $\mathrm{S} 5 . \dagger$

\section{Discussion}

Most commonly consumed foods in Western diets are low in dietary fiber and contribute to the high risk of many chronic
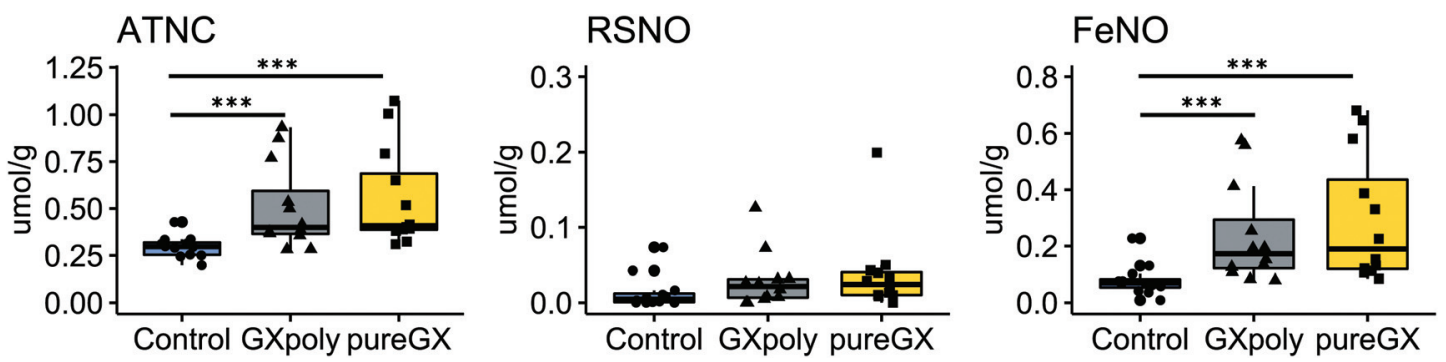

Fig. 3 The concentrations of nitroso compounds in the colonic contents of the control, GXpoly, and pureGX groups ( $\mu$ mol $g^{-1}$ of fecal weight). Concentrations of ATNC and FeNO were significantly higher in the GX groups than in the control group. There was no statistically significant difference in RSNO concentration. (Two-way ANOVA, ${ }^{*} p \leq 0.05,{ }^{* *} p \leq 0.01,{ }^{* * *} p \leq 0.001$, ATNC $=$ apparent total N-nitroso compounds, FeNO $=$ nitrosyl iron, RSNO = nitrosothiols, GXpoly = glucuronoxylan- and polyphenol-rich hemicellulose extract, pureGX = highly purified glucuronoxylan-rich hemicellulose extract). 
A
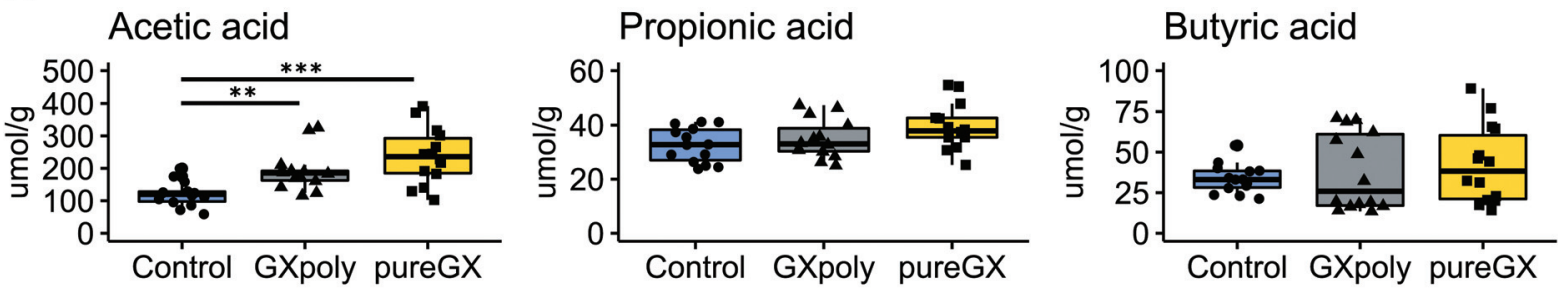

BCFA

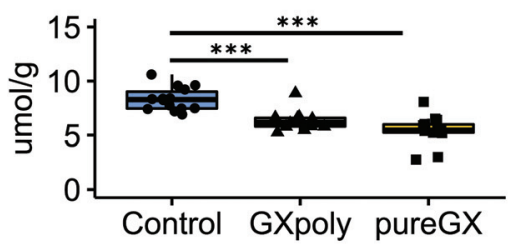

B

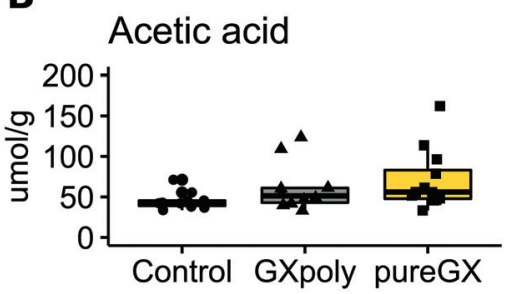

BCFA

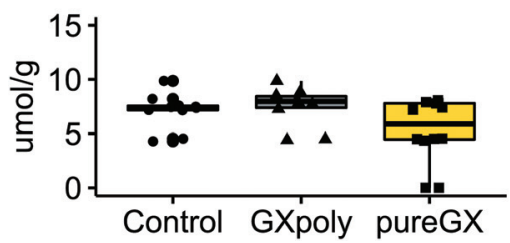

Total SCFAs

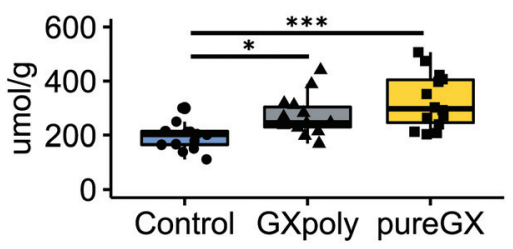

Propionic acid
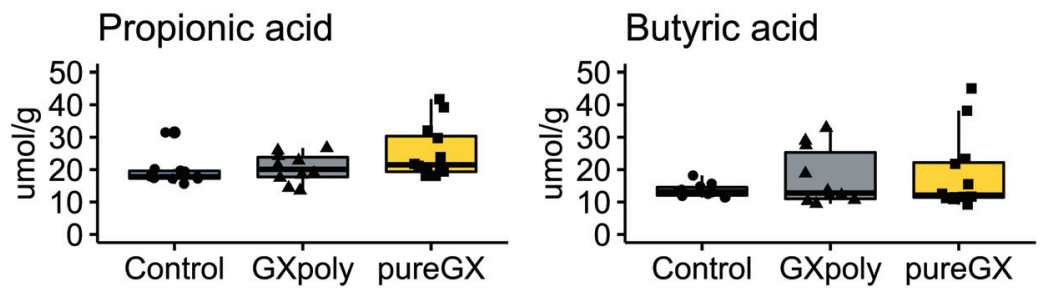

Fig. 4 Cecal and distal colonic concentrations ( $\mu \mathrm{mol} \mathrm{g}{ }^{-1}$ of dry weight) of SCFAs in Wistar rats fed control, GXpoly and pureGX diets. (A) Cecal total SCFA $(p<0.001)$ and acetate $(p<0.001)$ concentrations $(n=14$ per group) were higher in the GX groups than in the control group. (B) Colonic SCFA concentrations (control $n=10$, pureGX $n=12$, GXpoly $n=10$ ) did not differ between the groups even though the trends were similar to that of the cecal samples (two-way ANOVA, ${ }^{*} p \leq 0.05,{ }^{* *} p \leq 0.01,{ }^{* *} p \leq 0.001, \mathrm{BCFA}=$ branched-chain fatty acid, SCFA $=$ short-chain fatty acid, GXpoly $=$ glucuronoxylan- and polyphenol-rich hemicellulose extract, pureGX = highly purified glucuronoxylan-rich hemicellulose extract).

diseases, including colorectal cancer. ${ }^{57}$ Thus, there is a need to incorporate more fiber-rich foods into diets in general. As excellent food stabilizers, GX-rich extracts would be a straightforward way to increase the dietary fiber content of foods. However, it has remained unclear, how they interact in the colon. Here, we studied the effects of two differently processed birch-derived GX-rich extracts with different levels of polyphenol content on the gut microbiota, SCFAs, and NOCs in healthy Wistar rats. We found that GX consumption induced significant enrichment of bifidobacteria as well as other health-associated bacterial groups and increased colonic fermentation indicated by higher concentrations of bacterial metabolites and total quantity of bacteria.

Microbiota richness and alpha diversity, indices that reflect overall microbiota structure, were significantly lower in the GX groups than in the cellulose control group. While low microbiota richness and diversity are often associated with poor metabolic health, ${ }^{58}$ in the present study they likely resulted from the selective promotion of potentially beneficial bacteria, such as Bifidobacteriaceae and Lactobacillaceae. Similar observations have been reported in a recent intervention studying dietary fibers in individuals with type 2 diabetes. ${ }^{59}$ Our study demonstrates that GX containing diets have prebiotic effects. Both GXpoly and pureGX diets supported the growth of the probiotic Bifidobacteriaceae as indicated by its significantly higher relative and absolute abundance. This is in line with previous studies demonstrating that wood- and cereal-derived XOS display bifidogenic properties in vitro and in vivo, respectively. ${ }^{25,60,61}$ The bifidogenic effect of GX may be attributed to the potential ability of Bifidobacteriaceae to ferment GX, or trophic interactions with xylanolytic bacteria, such as Bacteroides, ${ }^{62}$ which had significantly higher absolute abundance in the GX groups. Particularly pureGX also favored the growth of Lactobacillaceae, the absolute abundance being 
5 -fold higher in the pureGX group than in the control group. In contrast, the relative abundance of Lactobacillaceae failed to increase significantly in the GXpoly group. This difference between pureGX and GXpoly may be explained by the high purity of pureGX, which contains more hemicellulose that can be utilized by lactic acid bacteria, including Lactobacillaceae. ${ }^{63}$

The health-promoting effects of intestinal commensals, such as Bifidobacteriaceae and Lactobacillaceae, are thought to be mediated by the production of microbial metabolites including SCFAs. We found that acetic acid and total SCFAs were significantly elevated in the cecum of the rats on the GX diets, and the levels of individual SCFAs were strongly associated with their expected bacterial producers. Acetate is the most abundant SCFA in peripheral circulation with direct systemic effects. It is able to cross the blood-brain barrier and affect hypothalamic control of appetite, increasing satiety. ${ }^{64}$ It can also affect adipose tissue metabolism, improving overall metabolic health via $\mathrm{G}$ protein-coupled receptor $43 .^{23}$ PureGX increased the propionate concentration with borderline statistical significance. Propionate is gluconeogenic and may increase satiety by inducing postprandial GLP-1 and PYY secretion. $^{23}$ However, we did not notice difference in food intake between the groups, which may be explained by the use of young growing rats in our experiment. Both acetate and propionate can dampen pro-inflammatory cytokine production. ${ }^{65}$ Our findings are in line with previous in vitro studies where AGX had a propiogenic effect, ${ }^{18}$ and XOS increased the levels of acetate. ${ }^{24}$

Other bacterial groups that exhibited drastically higher relative and absolute abundances following the GX diets are Atopobiaceae, linked to positive cardiometabolic health, ${ }^{66}$ and butyrate-producing, leanness-associated Eisenbergiella. ${ }^{67,68}$ In agreement with our findings, previous in vivo and in vitro studies have reported the increases in those bacteria by the supplementation of several types of complex dietary carbohydrates, such as wood-derived acetylated galactoglucomannan and inulin. ${ }^{18,66}$ It is worth noting that Bacteroidaceae and leanness-associated Christensenellaceae ${ }^{69}$ had higher absolute abundances, but not relative abundances, in the GX groups. This discrepancy is likely due to compositionality effects that may lead to erroneous interpretations of the findings if only relative abundance was analyzed. ${ }^{51,70}$

It is noteworthy that the GX diets decreased the relative and absolute abundances of the bacterial groups implicated in proteolytic fermentation, Peptostreptococcaceae, and Peptococcaceae, which have been linked to obesity and intestinal inflammation. ${ }^{71}$ A previous mouse study also documented the reduction in Peptostreptococcaceae and Peptococcaceae following a diet enriched in cranberry beans that contain abundant phenolic compounds and nondigestible fermentable components. ${ }^{72}$ However, in our study the abundance of proteolytic bacteria was the same in the polyphenol-rich GXpoly and pureGX groups, which indicates that the lower abundances of those bacteria in GX groups are due to the structural differences between the GX and cellulose, not the polyphenols. The abundance of proteolytic bacteria was correlated with the con- centrations of BCFAs, which were lower in the GX groups than in the control group. This is in accordance with a previous study, where wheat arabinoxylan-oligosaccharide supplementation lowered fecal BCFA content in children. ${ }^{34}$ Bifidobacteria have been shown to decrease BCFA concentration, ${ }^{73}$ which may have contributed to the difference in the cecal BCFAs between the dietary groups. Moreover, a higher abundance of fermentable fibers in GX groups may have reduced the relative protein availability for bacterial fermentation. BCFAs are considered a biomarker of protein catabolism ${ }^{65}$ and they have been linked to increased hepatic insulin resistance. ${ }^{74}$ Nevertheless, little is known regarding the impact of BCFAs on host health to date.

On the other hand, GX increased the concentrations of ATNC and FeNO that are nitrosated from amines produced by microbial fermentation of proteins. ${ }^{30}$ NOCs are potentially carcinogenic, ${ }^{30}$ but limited dietary studies have reported their concentrations. It has previously been noted that high concentrations of fecal NOCs are related to a high intake of red meat. ${ }^{75}$ However, in a recent study inulin was able to attenuate the formation of ATNC and FeNO in the gut of healthy rats fed processed meat. ${ }^{32}$ Especially some facultative anaerobes, such as Escherichia coli, have been linked to increased NOC formation in vitro. ${ }^{76}$ However, we did not notice a difference in known NOC-forming bacteria abundance between the dietary groups, and thus the higher levels of ATNC and FeNO in the GX groups presumably resulted from the elevated overall nitrate-reducing capacities arising from the increased total bacterial population, i.e., increased colonic fermentation.

The relative abundance of sulfidogenic, pro-inflammatory, and lipopolysaccharide (LPS)-producing Bilophila ${ }^{77}$ was lower in the GX groups than in the control group. LPS is one of the most potent pathogen-associated molecular patterns that elicits innate immune responses, ${ }^{78}$ and also the predicted functions related to its biosynthesis were reduced following the GX diets. This is in line with a previous intervention study where another fermentable fiber, inulin, decreased relative abundance of Bilophila. ${ }^{79}$ Additionally, GXpoly specifically decreased abundance of Akkermansia, which is a mucolytic commensal linked to improved metabolism, ${ }^{80}$ but also shown to promote the colonization of mucosal pathogens in the context of fiber deprivation. ${ }^{81}$ While several studies have reported that the abundance of Akkermansia was elevated by polyphenols, ${ }^{82}$ the reduced Akkermansia abundance observed in the polyphenol-rich GXpoly group is likely attributable to the structural differences in the heterogeneous phenolic compounds. Previous animal studies using diets rich in polyphenolic lignans similarly found a large decrease in Akkermansia following the treatment. ${ }^{83,84}$

In addition to microbiota modulation and production of SCFAs, dietary fibers can affect health via altering gut function and stool properties. ${ }^{57}$ Certain dietary fibers can protect from constipation and diarrhea, e.g., provide regularity, which can be evaluated by measuring fecal water content, and fecal weight per day. ${ }^{85}$ Fecal water content is dependent 
on the ability of dietary fiber to bind water or stimulate water secretion in the gut, ${ }^{85}$ and it correlates with fecal weight. ${ }^{86}$ In our study, the fecal water content was the same across the groups, but the fecal weight was higher in the cellulose group than in the GX groups, suggesting that GX and cellulose increased water content through different mechanisms. ${ }^{86}$ The result is in line with previous findings where less fermentable fibers have been shown to have a more significant effect on fecal weight than more fermentable fibers. ${ }^{87}$ However, the difference did not reach statistical significance when cellulose was compared to the GXpoly group. This difference between GXpoly and pureGX diets is probably due to lignin that was measured with a Pyr-GC/MS. The method can be used to estimate the lignin content of hemicelluloses ${ }^{5}$ hence having a potential as an easy method to define polyphenol content of dietary fibers. We detected lignin in the GXpoly but not in the pureGX diet, although it is assumed that the pureGX diet contains polyphenolic compounds below the detection limit of Pyr-GC/MS. The result indicates that lignin of the GXpoly diet provided bulking and was not broken down by the gut microbiota. However, as demonstrated in previous studies, gut microbiota may utilize lignin to form lignans, e.g. enterolactone and enterodiol, ${ }^{13,15,16}$ which have been shown to have a protective effect against colorectal cancer. ${ }^{14}$ Some of the lignin in GXpoly diet may have been degraded into lignans indicated by the abundance of polyphenol degrading bacteria, Eggerthellaceae, in the GXpoly group. Clearly, the effects of birch-derived lignin on fecal properties and bacterial metabolites needs to be further studied.

The feeding of GX-rich extract for four weeks did not cause adverse effects in any of the animals, which can be considered a positive sign for the safe use of the extract. In a recent study glucuronoxylan-rich extract was shown not to be toxic to normal human cells, which supports our findings. ${ }^{88}$ Furthermore, we did not notice a statistically significant difference between the treated and control group in food and water intake, which indicates that GX does not interfere with these parameters or reduce palatability. Body weight of the rats eating the GXpoly or pureGX diet did not differ from the control group throughout the study, suggesting that GXpoly or pureGX does not compromise nutrient absorption. Nevertheless, to prove that GX is non-toxic in the long run further studies are needed to enlighten the physiological effects of the extracts.

While the effect of sex on NOCs has not been previously reported, sex represents an important factor affecting the concentrations of acetate and NOCs in the present study. We also noticed a sex difference in some bacterial taxa. However, as those bacteria are not known for enzymes responsible for a nitrosation reaction, and acetate can be produced by many gut bacteria, the finding does not directly explain the difference in microbial metabolites between male and female rats. It has been shown that sex impacts the metabolism of dietary fiber, and thus potential health effects of functional foods should be studied on males and females. ${ }^{89}$
The main strength of our report is the in vivo study setting representing relevant physiological conditions. Several host physiological factors, such as immunity, ${ }^{90}$ lipid metabolism, ${ }^{91}$ and intestinal transit time, ${ }^{92}$ all have been reported to influence the effects of xylan on the gut microbiota and cannot be inferred from the existing in vitro studies. Quantitative microbiome profiling providing information on absolute abundances of the gut microbes that would be otherwise overlooked represents another strength in the present study. While the characterization of the gut microbiota was limited in resolution due to the nature of $16 \mathrm{~S}$ rRNA gene amplicon sequencing, we compensated for this limitation by using imputed metagenomic analysis. As the increases in SCFAs may have different implications depending on host health states, future studies should be performed in animal models with certain metabolic phenotypes and/or on specific diets to explore the potential benefits or anti-inflammatory effects of the observations made in the present study. In addition, other GXpoly and pureGX derived metabolites can participate in potential beneficial and anti-inflammatory effects, which should be elaborated in future unbiased metabolomic studies. Lastly, the inferred health effects of GX-associated bacteria discussed above are mainly from human studies. Hence, human trials are needed to evaluate the translatability of our findings before birch GX can be used in foods.

\section{Conclusion}

In summary, our results suggest potential health benefits of GX via supporting the growth of beneficial bacteria and suppressing the detrimental ones. Consistently both GXpoly and pureGX also supported the production of potentially beneficial SCFAs and diminished the production of potentially harmful BCFAs. Despite the higher fermentability, the extracts, especially GXpoly, had similar favorable effects on fecal properties as cellulose. Taken together, these findings suggest that birch-derived GX extracts that can be utilized as ecological food stabilizers are able to alter the gut microbiota and colonic fermentation in vivo, potentially towards improved gut health.

\section{Author contributions}

EK: Investigation, formal analysis, writing - original draft, visualization; ML: Investigation, methodology, validation, visualization; CJ: Investigation, formal analysis, writing - original draft, visualization; AS: Supervision; TH: Methodology; KM: Conceptualization, supervision, funding acquisition, writing review \& editing; AMP: Conceptualization, supervision, writing - review \& editing.

\section{Conflicts of interest}

The authors declare that there is no conflict of interest. 


\section{Acknowledgements}

We are grateful to Ms Anu Heiman-Lindh for her help in carrying out the animal experiment and collecting samples, to $\mathrm{Mr}$ Miikka Olin for his assistance with gas chromatography data acquisition, to Mr Troy Faithfull for editing the manuscript, and to Petri Kilpeläinen and Lari Vähäsalo for providing the glucuronoxylan extracts. We thank Evgenia Dikareva for performing the qPCR assays for total bacterial quantification. This work was supported by Jane and Aatos Erkko Foundation and by the University of Helsinki starting grant for a new tenure track professor in food sciences.

\section{References}

1 E. Capuano, The behavior of dietary fiber in the gastrointestinal tract determines its physiological effect, Crit. Rev. Food Sci. Nutr., 2017, 57, 3543-3564.

2 A. M. Stephen, M. M.-J. Champ, S. J. Cloran, M. Fleith, L. van Lieshout, H. Mejborn and V. J. Burley, Dietary fibre in Europe: current state of knowledge on definitions, sources, recommendations, intakes and relationships to health, Nutr. Res. Rev., 2017, 30, 149-190.

3 L. Pitkänen, M. Heinonen and K. S. Mikkonen, Safety considerations of plant polysaccharides for food use: a case study on phenolic-rich softwood galactoglucomannan extract, Food Funct., 2018, 9, 1931-1943.

4 E. Sjöstrom, Wood Chemistry: Fundamentals and Applications, Academic Press, San Diego, 1993.

5 M. H. Lahtinen, F. Valoppi, V. Juntti, S. Heikkinen, P. O. Kilpeläinen, N. H. Maina and K. S. Mikkonen, LigninRich PHWE Hemicellulose Extracts Responsible for Extended Emulsion Stabilization, Front. Chem., 2019, 7, 871.

6 S. Kirjoranta, A. Knaapila, P. Kilpeläinen and K. S. Mikkonen, Sensory profile of hemicellulose-rich wood extracts in yogurt models, Cellulose, 2020, 27, 7607-7620.

7 A. Fadel, A. M. Mahmoud, J. J. Ashworth, W. Li, Y. L. Ng and A. Plunkett, Health-related effects and improving extractability of cereal arabinoxylans, Int. J. Biol. Macromol., 2018, 109, 819-831.

8 P. V. P. Nirmala and I. J. Joye, Dietary Fibre from Whole Grains and Their Benefits on Metabolic Health, Nutrients, 2020, 12, 3045.

9 K. S. Mikkonen, C. Laine, I. Kontro, R. A. Talja, R. Serimaa and M. Tenkanen, Combination of internal and external plasticization of hydroxypropylated birch xylan tailors the properties of sustainable barrier films, Eur. Polym. J., 2015, 66, 307-318.

10 F. Valoppi, M. H. Lahtinen, M. Bhattarai, S. J. Kirjoranta, V. K. Juntti, L. J. Peltonen, P. O. Kilpeläinen and K. S. Mikkonen, Centrifugal fractionation of softwood extracts improves the biorefinery workflow and yields functional emulsifiers, Green Chem., 2019, 21, 4691-4705.
11 D. M. de Carvalho, M. H. Lahtinen, M. Lawoko and K. S. Mikkonen, Enrichment and Identification of LigninCarbohydrate Complexes in Softwood Extract, ACS Sustainable Chem. Eng., 2020, 8, 11795-11804.

12 J. A. Monro and S. Mishra, Digestion-Resistant Remnants of Vegetable Vascular and Parenchyma Tissues Differ in Their Effects in the Large Bowel of Rats, Food Dig., 2010, 1, 47-56.

13 A. N. Begum, C. Nicolle, I. Mila, C. Lapierre, K. Nagano, K. Fukushima, S.-M. Heinonen, H. Adlercreutz, C. Rémésy and A. Scalbert, Dietary lignins are precursors of mammalian lignans in rats, J. Nutr., 2004, 134, 120-127.

14 J. M. Landete, Plant and mammalian lignans: A review of source, intake, metabolism, intestinal bacteria and health, Food Res. Int., 2012, 46, 410-424.

15 P. Niemi, A.-M. Aura, J. Maukonen, A. I. Smeds, I. Mattila, K. Niemelä, T. Tamminen, C. B. Faulds, J. Buchert and K. Poutanen, Interactions of a Lignin-Rich Fraction from Brewer's Spent Grain with Gut Microbiota in Vitro, J. Agric. Food Chem., 2013, 61, 6754-6762.

16 A.-M. Aura, P. Niemi, I. Mattila, K. Niemelä, A. Smeds, T. Tamminen, C. Faulds, J. Buchert and K. Poutanen, Release of Small Phenolic Compounds from Brewer's Spent Grain and Its Lignin Fractions by Human Intestinal Microbiota in Vitro, J. Agric. Food Chem., 2013, 61, 9744-9753.

17 K. Makki, E. C. Deehan, J. Walter and F. Bäckhed, The Impact of Dietary Fiber on Gut Microbiota in Host Health and Disease, Cell Host Microbe, 2018, 23, 705-715.

18 S. L. La Rosa, V. Kachrimanidou, F. Buffetto, P. B. Pope, N. A. Pudlo, E. C. Martens, R. A. Rastall, G. R. Gibson and B. Westereng, Wood-Derived Dietary Fibers Promote Beneficial Human Gut Microbiota, mSphere, 2019, 4, e00554-e00518.

19 L. Polari, P. Ojansivu, S. Mäkelä, C. Eckerman, B. Holmbom and S. Salminen, Galactoglucomannan Extracted from Spruce (Picea abies) as a Carbohydrate Source for Probiotic Bacteria, J. Agric. Food Chem., 2012, 60, 11037-11043.

20 V. Deloule, C. Boisset, D. Hannani, A. Suau, A. Le Gouellec, J. Chroboczek, C. Botté, Y. Yamaryo-Botté, C. Chirat and B. Toussaint, Prebiotic role of softwood hemicellulose in healthy mice model, J. Funct. Foods, 2020, 64, 103688.

21 Y. Konkol, A. Keskitalo, H. Vuorikoski, S. Pietilä, L. L. Elo, E. Munukka, J. Bernoulli and J. Tuomela, Chronic nonbacterial prostate inflammation in a rat model is associated with changes of gut microbiota that can be modified with a galactoglucomannan-rich hemicellulose extract in the diet, BJU Int., 2019, 123, 899-908.

22 Y. Wu, Y. Chen, Y. Lu, H. Hao, J. Liu and R. Huang, Structural features, interaction with the gut microbiota and anti-tumor activity of oligosaccharides, RSC Adv., 2020, 10, 16339-16348.

23 A. Koh, F. De Vadder, P. Kovatcheva-Datchary and F. Bäckhed, From Dietary Fiber to Host Physiology: ShortChain Fatty Acids as Key Bacterial Metabolites, Cell, 2016, 165, 1332-1345. 
24 M. A. Kabel, L. Kortenoeven, H. A. Schols and A. G. J. Voragen, In vitro fermentability of differently substituted xylo-oligosaccharides, J. Agric. Food Chem., 2002, 50, 6205-6210.

25 P. Moura, S. Cabanas, P. Lourenço, F. Gírio, M. C. LoureiroDias and M. P. Esteves, In vitro fermentation of selected xylo-oligosaccharides by piglet intestinal microbiota, $L W T$ Food Sci. Technol., 2008, 41, 1952-1961.

26 J. L. Carlson, J. M. Erickson, J. M. Hess, T. J. Gould and J. L. Slavin, Prebiotic Dietary Fiber and Gut Health: Comparing the in Vitro Fermentations of Beta-Glucan, Inulin and Xylooligosaccharide, Nutrients, 2017, 9, 1361.

27 J. M. Campbell, G. C. Fahey Jr. and B. W. Wolf, Selected Indigestible Oligosaccharides Affect Large Bowel Mass, Cecal and Fecal Short-Chain Fatty Acids, pH and Microflora in Rats, J. Nutr., 1997, 127, 130-136.

28 J. Long, J. Yang, S. M. Henning, S. L. Woo, M. Hsu, B. Chan, D. Heber and Z. Li, Xylooligosaccharide supplementation decreases visceral fat accumulation and modulates cecum microbiome in mice, J. Funct. Foods, 2019, 52, 138-146.

29 P. Thomson, D. A. Medina, V. Ortúzar, M. Gotteland and D. Garrido, Anti-inflammatory effect of microbial consortia during the utilization of dietary polysaccharides, Food Res. Int., 2018, 109, 14-23.

30 R. Hughes and I. R. Rowland, Metabolic Activities of the Gut Microflora in Relation to Cancer, Microb. Ecol. Health Dis., 2000, 12, 179-185.

31 G. T. Macfarlane and S. Macfarlane, Bacteria, Colonic Fermentation, and Gastrointestinal Health, J. AOAC Int., 2012, 95, 50-60.

32 R. Thøgersen, N. Gray, G. Kuhnle, T. Van Hecke, S. De Smet, J. F. Young, U. K. Sundekilde, A. K. Hansen and H. C. Bertram, Inulin-fortification of a processed meat product attenuates formation of nitroso compounds in the gut of healthy rats, Food Chem., 2020, 302, 125339.

33 M. H. Lewin, N. Bailey, T. Bandaletova, R. Bowman, A. J. Cross, J. Pollock, D. E. G. Shuker and S. A. Bingham, Red Meat Enhances the Colonic Formation of the DNA Adduct O6-Carboxymethyl Guanine: Implications for Colorectal Cancer Risk, Cancer Res., 2006, 66, 1859-1865.

34 I. E. J. A. François, O. Lescroart, W. S. Veraverbeke, M. Marzorati, S. Possemiers, H. Hamer, K. Windey, G. W. Welling, J. A. Delcour, C. M. Courtin, K. Verbeke and W. F. Broekaert, Effects of wheat bran extract containing arabinoxylan oligosaccharides on gastrointestinal parameters in healthy preadolescent children, J. Pediatr. Gastroenterol. Nutr., 2014, 58, 647-653.

35 M. Centanni, J. C. Hutchison, S. M. Carnachan, A. M. Daines, W. J. Kelly, G. W. Tannock and I. M. Sims, Differential growth of bowel commensal Bacteroides species on plant xylans of differing structural complexity, Carbohydr. Polym., 2017, 157, 1374-1382.

36 D. J. Rose, J. A. Patterson and B. R. Hamaker, Structural Differences among Alkali-Soluble Arabinoxylans from Maize (Zea mays), Rice (Oryza sativa), and Wheat (Triticum aestivum) Brans Influence Human Fecal Fermentation Profiles, J. Agric. Food Chem., 2010, 58, 493-499.

37 P. O. Kilpeläinen, S. S. Hautala, O. O. Byman, L. J. Tanner, R. I. Korpinen, M. K.-J. Lillandt, A. V. Pranovich, V. H. Kitunen, S. M. Willför and H. S. Ilvesniemi, Pressurized hot water flow-through extraction system scale up from the laboratory to the pilot scale, Green Chem., 2014, 16, 3186-3194.

38 S. Von Schoultz, US Pat., 16796898, 2020.

39 K. S. Mikkonen, S. Kirjoranta, C. Xu, J. Hemming, A. Pranovich, M. Bhattarai, L. Peltonen, P. Kilpeläinen, N. Maina, M. Tenkanen, M. Lehtonen and S. Willför, Environmentally-compatible alkyd paints stabilized by wood hemicelluloses, Ind. Crops Prod., 2019, 133, 212-220.

40 C. Kilkenny, W. Browne, I. C. Cuthill, M. Emerson and D. G. Altman, Animal research: Reporting in vivo experiments: The ARRIVE guidelines, Br. J. Pharmacol., 2010, 160, 1577-1579.

41 S. Getahun, S. Septien, J. Mata, T. Somorin, I. Mabbett and C. Buckley, Drying characteristics of faecal sludge from different on-site sanitation facilities, J. Environ. Manage., 2020, 261, 110267.

42 A. Salonen, J. Nikkilä, J. Jalanka-Tuovinen, O. Immonen, M. Rajilić-Stojanović, R. A. Kekkonen, A. Palva and W. M. de Vos, Comparative analysis of fecal DNA extraction methods with phylogenetic microarray: effective recovery of bacterial and archaeal DNA using mechanical cell lysis, J. Microbiol. Methods, 2010, 81, 127-134.

43 K. Korpela, A. Salonen, H. Saxen, A. Nikkonen, V. Peltola, T. Jaakkola, W. de Vos and K.-L. Kolho, Antibiotics in early life associate with specific gut microbiota signatures in a prospective longitudinal infant cohort, Pediatr. Res., 2020, 88, 438-443.

44 E. Bolyen, J. R. Rideout, M. R. Dillon, N. A. Bokulich, C. C. Abnet, G. A. Al-Ghalith, H. Alexander, E. J. Alm, M. Arumugam, F. Asnicar, Y. Bai, J. E. Bisanz, K. Bittinger, A. Brejnrod, C. J. Brislawn, C. T. Brown, B. J. Callahan, A. M. Caraballo-Rodríguez, J. Chase, E. K. Cope, R. Da Silva, C. Diener, P. C. Dorrestein, G. M. Douglas, D. M. Durall, C. Duvallet, C. F. Edwardson, M. Ernst, M. Estaki, J. Fouquier, J. M. Gauglitz, S. M. Gibbons, D. L. Gibson, A. Gonzalez, K. Gorlick, J. Guo, B. Hillmann, S. Holmes, H. Holste, C. Huttenhower, G. A. Huttley, S. Janssen, A. K. Jarmusch, L. Jiang, B. D. Kaehler, K. B. Kang, C. R. Keefe, P. Keim, S. T. Kelley, D. Knights, I. Koester, T. Kosciolek, J. Kreps, M. G. I. Langille, J. Lee, R. Ley, Y.-X. Liu, E. Loftfield, C. Lozupone, M. Maher, C. Marotz, B. D. Martin, D. McDonald, L. J. McIver, A. V. Melnik, J. L. Metcalf, S. C. Morgan, J. T. Morton, A. T. Naimey, J. A. Navas-Molina, L. F. Nothias, S. B. Orchanian, T. Pearson, S. L. Peoples, D. Petras, M. L. Preuss, E. Pruesse, L. B. Rasmussen, A. Rivers, M. S. Robeson, P. Rosenthal, N. Segata, M. Shaffer, A. Shiffer, R. Sinha, S. J. Song, J. R. Spear, A. D. Swafford, L. R. Thompson, P. J. Torres, P. Trinh, A. Tripathi, P. J. Turnbaugh, S. Ul-Hasan, J. J. J. van der Hooft, F. Vargas, Y. Vázquez-Baeza, E. Vogtmann, M. von 
Hippel, W. Walters, Y. Wan, M. Wang, J. Warren, K. C. Weber, C. H. D. Williamson, A. D. Willis, Z. Z. Xu, J. R. Zaneveld, Y. Zhang, Q. Zhu, R. Knight and J. G. Caporaso, Reproducible, interactive, scalable and extensible microbiome data science using QIIME 2, Nat. Biotechnol., 2019, 37, 852-857.

45 B. J. Callahan, P. J. McMurdie, M. J. Rosen, A. W. Han, A. J. A. Johnson and S. P. Holmes, DADA2: High-resolution sample inference from Illumina amplicon data, Nat. Methods, 2016, 13, 581-583.

46 C. Quast, E. Pruesse, P. Yilmaz, J. Gerken, T. Schweer, P. Yarza, J. Peplies and F. O. Glöckner, The SILVA ribosomal RNA gene database project: improved data processing and web-based tools, Nucleic Acids Res., 2013, 41, D590-D596.

47 M. Kanehisa, S. Goto, Y. Sato, M. Furumichi and M. Tanabe, KEGG for integration and interpretation of large-scale molecular data sets, Nucleic Acids Res., 2012, 40, D109-D114.

48 G. M. Douglas, V. J. Maffei, J. R. Zaneveld, S. N. Yurgel, J. R. Brown, C. M. Taylor, C. Huttenhower and M. G. I. Langille, PICRUSt2 for prediction of metagenome functions, Nat. Biotechnol., 2020, 38, 685-688.

49 K. Korpela: Microbiota Analysis in $\mathrm{R}$ Easily. $\mathrm{R}$ package version 1.0, 2016, https:/github.com/katrikorpela/mare, (accessed 14 June 2021).

50 J. Oksanen, F. G. Blanchet, M. Friendly, R. Kindt, P. Legendre, D. McGlinn, P. R. Minchin, R. B. O’Hara, G. L. Simpson, P. Solymos, M. H. H. Stevens, E. Szoecs and H. Wagner, vegan, 2020, https:/CRAN.R-project.org/ package=vegan, (accessed 14 June 2021).

51 C. Jian, P. Luukkonen, H. Yki-Järvinen, A. Salonen and K. Korpela, Quantitative PCR provides a simple and accessible method for quantitative microbiota profiling, PLoS One, 2020, 15, e0227285.

52 G. G. C. Kuhnle, G. W. Story, T. Reda, A. R. Mani, K. P. Moore, J. C. Lunn and S. A. Bingham, Diet-induced endogenous formation of nitroso compounds in the GI tract, Free Radicals Biol. Med., 2007, 43, 1040-1047.

53 S. Chaberek, A. E. Frost, M. A. Doran and N. J. Bicknell, Interaction of some divalent metal ions with diethylenetriaminepentaacetic acid, J. Inorg. Nucl. Chem., 1959, 11, 184-196.

54 M. Feelisch, T. Rassaf, S. Mnaimneh, N. Singh, N. S. Bryan, D. Jourd'Heuil and M. Kelm, Concomitant S-, N-, and hemenitros(yl)ation in biological tissues and fluids: implications for the fate of NO in vivo, FASEB J., 2002, 16, 1775-1785.

55 H. Wang, Y. Liu, J. Shao, Y. Luo, W. Cai and L. Chen, Rapid and Accurate Simultaneous Determination of Seven ShortChain Fatty Acids in Feces by Gas Chromatography - Mass Spectrometry (GC-MS): Application in Type 2 Diabetic Rats and Drug Therapy, Anal. Lett., 2020, 53, 2320-2336.

56 G. Zhao, M. Nyman and J. A. Jönsson, Rapid determination of short-chain fatty acids in colonic contents and faeces of humans and rats by acidified water-extraction and directinjection gas chromatography, Biomed. Chromatogr., 2006, 20, 674-682.
57 D. Aune, D. S. M. Chan, R. Lau, R. Vieira, D. C. Greenwood, E. Kampman and T. Norat, Dietary fibre, whole grains, and risk of colorectal cancer: systematic review and dose-response meta-analysis of prospective studies, BMJ, 2011, 343, d6617.

58 J. L. Sonnenburg and F. Bäckhed, Diet-microbiota interactions as moderators of human metabolism, Nature, 2016, 535, 56-64.

59 L. Zhao, F. Zhang, X. Ding, G. Wu, Y. Y. Lam, X. Wang, H. Fu, X. Xue, C. Lu, J. Ma, L. Yu, C. Xu, Z. Ren, Y. Xu, S. Xu, H. Shen, X. Zhu, Y. Shi, Q. Shen, W. Dong, R. Liu, Y. Ling, Y. Zeng, X. Wang, Q. Zhang, J. Wang, L. Wang, Y. Wu, B. Zeng, H. Wei, M. Zhang, Y. Peng and C. Zhang, Gut bacteria selectively promoted by dietary fibers alleviate type 2 diabetes, Science, 2018, 359, 1151-1156.

60 D. Gobinath, A. N. Madhu, G. Prashant, K. Srinivasan and S. G. Prapulla, Beneficial effect of xylo-oligosaccharides and fructo-oligosaccharides in streptozotocin-induced diabetic rats, Br. J. Nutr., 2010, 104, 40-47.

61 M. Nieto-Domínguez, L. I. de Eugenio, M. J. York-Durán, B. Rodríguez-Colinas, F. J. Plou, E. Chenoll, E. Pardo, F. Codoñer and M. J. Martínez, Prebiotic effect of xylooligosaccharides produced from birchwood xylan by a novel fungal GH11 xylanase, Food Chem., 2017, 232, 105-113.

62 C. Chassard, V. Goumy, M. Leclerc, C. Del'homme and A. Bernalier-Donadille, Characterization of the xylandegrading microbial community from human faeces, FEMS Microbiol. Ecol., 2007, 61, 121-131.

63 L. Tarraran and R. Mazzoli, Alternative strategies for lignocellulose fermentation through lactic acid bacteria: the state of the art and perspectives, FEMS Microbiol. Lett., 2018, 365, fny126.

64 G. Frost, M. L. Sleeth, M. Sahuri-Arisoylu, B. Lizarbe, S. Cerdan, L. Brody, J. Anastasovska, S. Ghourab, M. Hankir, S. Zhang, D. Carling, J. R. Swann, G. Gibson, A. Viardot, D. Morrison, E. L. Thomas and J. D. Bell, The short-chain fatty acid acetate reduces appetite via a central homeostatic mechanism, Nat. Commun., 2014, 5, 3611.

65 K. Oliphant and E. Allen-Vercoe, Macronutrient metabolism by the human gut microbiome: major fermentation by-products and their impact on host health, Microbiome, 2019, 7, 91.

66 P. C. Vinke, S. El Aidy and G. van Dijk, The Role of Supplemental Complex Dietary Carbohydrates and Gut Microbiota in Promoting Cardiometabolic and Immunological Health in Obesity: Lessons from Healthy Non-Obese Individuals, Front. Nutr., 2017, 4, 34.

67 I. Amir, P. Bouvet, C. Legeay, U. Gophna and A. Weinberger, Eisenbergiella tayi gen. nov., sp. nov., isolated from human blood, Int. J. Syst. Evol. Microbiol., 2014, 64, 907-914.

68 Z. Jie, X. Yu, Y. Liu, L. Sun, P. Chen, Q. Ding, Y. Gao, X. Zhang, M. Yu, Y. Liu, Y. Zhang, K. Kristiansen, H. Jia, S. Brix and K. Cai, The Baseline Gut Microbiota Directs Dieting-Induced Weight Loss Trajectories, Gastroenterology, 2021, 160, 2029-2042.e16.

69 J. L. Waters and R. E. Ley, The human gut bacteria Christensenellaceae are widespread, heritable, and associated with health, BMC Biol., 2019, 17, 83. 
70 V. Lloréns-Rico, S. Vieira-Silva, P. J. Gonçalves, G. Falony and J. Raes, Benchmarking microbiome transformations favors experimental quantitative approaches to address compositionality and sampling depth biases, Nat. Commun., 2021, 12, 3562.

71 S. F. Clarke, E. F. Murphy, O. O’Sullivan, R. P. Ross, P. W. O'Toole, F. Shanahan and P. D. Cotter, Targeting the microbiota to address diet-induced obesity: a time dependent challenge, PLoS One, 2013, 8, e65790.

72 J. M. Monk, D. Lepp, C. P. Zhang, W. Wu, L. Zarepoor, J. T. Lu, K. P. Pauls, R. Tsao, G. A. Wood, L. E. Robinson and K. A. Power, Diets enriched with cranberry beans alter the microbiota and mitigate colitis severity and associated inflammation, J. Nutr. Biochem., 2016, 28, 129-139.

73 H. Mäkeläinen, S. Forssten, M. Saarinen, J. Stowell, N. Rautonen and A. C. Ouwehand, Xylo-oligosaccharides enhance the growth of bifidobacteria and Bifidobacterium lactis in a simulated colon model, Benefic. Microbes, 2010, 1, 81-91.

74 B. S.-Y. Choi, N. Daniel, V. P. Houde, A. Ouellette, B. Marcotte, T. V. Varin, C. Vors, P. Feutry, O. Ilkayeva, M. Ståhlman, P. St-Pierre, F. Bäckhed, A. Tremblay, P. J. White and A. Marette, Feeding diversified protein sources exacerbates hepatic insulin resistance via increased gut microbial branched-chain fatty acids and mTORC1 signaling in obese mice, Nat. Commun., 2021, 12, 3377.

75 S. A. Bingham, B. Pignatelli, J. R. Pollock, A. Ellul, C. Malaveille, G. Gross, S. Runswick, J. H. Cummings and I. K. O'Neill, Does increased endogenous formation of $\mathrm{N}$-nitroso compounds in the human colon explain the association between red meat and colon cancer?, Carcinogenesis, 1996, 17, 515-523.

76 J. Kobayashi, Effect of diet and gut environment on the gastrointestinal formation of N-nitroso compounds: A review, Nitric Oxide - Biol. Chem., 2018, 73, 66-73.

77 J. M. Natividad, B. Lamas, H. P. Pham, M.-L. Michel, D. Rainteau, C. Bridonneau, G. da Costa, J. van Hylckama Vlieg, B. Sovran, C. Chamignon, J. Planchais, M. L. Richard, P. Langella, P. Veiga and H. Sokol, Bilophila wadsworthia aggravates high fat diet induced metabolic dysfunctions in mice, Nat. Commun., 2018, 9, 2802.

78 R. Bohan, X. Tianyu, Z. Tiantian, F. Ruonan, H. Hongtao, W. Qiong and S. Chao, Gut microbiota: a potential manipulator for host adipose tissue and energy metabolism, J. Nutr. Biochem., 2019, 64, 206-217.

79 D. Vandeputte, G. Falony, S. Vieira-Silva, J. Wang, M. Sailer, S. Theis, K. Verbeke and J. Raes, Prebiotic inulin-type fructans induce specific changes in the human gut microbiota, Gut, 2017, 66, 1968-1974.

80 H. Plovier, A. Everard, C. Druart, C. Depommier, M. Van Hul, L. Geurts, J. Chilloux, N. Ottman, T. Duparc, L. Lichtenstein, A. Myridakis, N. M. Delzenne, J. Klievink, A. Bhattacharjee, K. C. H. van der Ark, S. Aalvink, L. O. Martinez, M.-E. Dumas, D. Maiter, A. Loumaye, M. P. Hermans, J.-P. Thissen, C. Belzer, W. M. de Vos and P. D. Cani, A purified membrane protein from
Akkermansia muciniphila or the pasteurized bacterium improves metabolism in obese and diabetic mice, Nat. Med., 2017, 23, 107-113.

81 M. S. Desai, A. M. Seekatz, N. M. Koropatkin, N. Kamada, C. A. Hickey, M. Wolter, N. A. Pudlo, S. Kitamoto, N. Terrapon, A. Muller, V. B. Young, B. Henrissat, P. Wilmes, T. S. Stappenbeck, G. Núñez and E. C. Martens, A Dietary Fiber-Deprived Gut Microbiota Degrades the Colonic Mucus Barrier and Enhances Pathogen Susceptibility, Cell, 2016, 167, 1339-1353.e21.

82 A. M. Alves-Santos, C. S. A. Sugizaki, G. C. Lima and M. M. V. Naves, Prebiotic effect of dietary polyphenols: A systematic review, J. Funct. Foods, 2020, 74, 104169.

83 S.-Y. Cho, J. Kim, J. H. Lee, J. H. Sim, D.-H. Cho, I.-H. Bae, H. Lee, M. A. Seol, H. M. Shin, T.-J. Kim, D.-Y. Kim, S.-H. Lee, S. S. Shin, S.-H. Im and H.-R. Kim, Modulation of gut microbiota and delayed immunosenescence as a result of syringaresinol consumption in middle-aged mice, Sci. Rep., 2016, 6, 39026.

84 K. A. Power, D. Lepp, L. Zarepoor, J. M. Monk, W. Wu, R. Tsao and R. Liu, Dietary flaxseed modulates the colonic microenvironment in healthy $\mathrm{C} 57 \mathrm{Bl} / 6$ male mice which may alter susceptibility to gut-associated diseases, J. Nutr. Biochem., 2016, 28, 61-69.

$85 \mathrm{~J}$. W. McRorie and N. M. McKeown, Understanding the Physics of Functional Fibers in the Gastrointestinal Tract: An Evidence-Based Approach to Resolving Enduring Misconceptions about Insoluble and Soluble Fiber, J. Acad. Nutr. Diet., 2017, 117, 251-264.

86 R. Spiller and L. Marciani, Intraluminal Impact of Food: New Insights from MRI, Nutrients, 2019, 11, 1145.

87 J. de Vries, A. Birkett, T. Hulshof, K. Verbeke and K. Gibes, Effects of Cereal, Fruit and Vegetable Fibers on Human Fecal Weight and Transit Time: A Comprehensive Review of Intervention Trials, Nutrients, 2016, 8, 130.

88 D. Granato, D. Reshamwala, R. Korpinen, L. Azevedo, M. A. Vieira do Carmo, T. M. Cruz, M. B. Marques, M. Wen, L. Zhang, V. Marjomäki and P. Kilpeläinen, From the forest to the plate - Hemicelluloses, galactoglucomannan, glucuronoxylan, and phenolic-rich extracts from unconventional sources as functional food ingredients, Food Chem., 2022, 381, 132284.

89 P. Shastri, J. McCarville, M. Kalmokoff, S. P. J. Brooks and J. M. Green-Johnson, Sex differences in gut fermentation and immune parameters in rats fed an oligofructose-supplemented diet, Biol. Sex Differ., 2015, 6, 13.

90 U. P. Tiwari, S. A. Fleming, M. S. A. Rasheed, R. Jha and R. N. Dilger, The role of oligosaccharides and polysaccharides of xylan and mannan in gut health of monogastric animals, J. Nutr. Sci., 2020, 9, e21.

91 M. Hashi and T. Takeshita, Hypocholesterolemic Effect of Beech (Fagus crenata Blume) Xylan on Cholesterol-fed Rats, Agric. Biol. Chem., 1975, 39, 579-583.

92 S. E. Fleming and B. Lee, Growth performance and intestinal transit time of rats fed purified and natural dietary fibers, J. Nutr., 1983, 113, 592-601. 